\title{
Modeling long-range cross-correlations in two-component ARFIMA and FIARCH processes
}

\author{
Boris Podobnik ${ }^{\mathrm{a}, \mathrm{b}}$ Davor Horvatic ${ }^{\mathrm{c}}$ Alfonso Lam $\mathrm{Ng}^{\mathrm{d}}$ \\ H. Eugene Stanley d Plamen Ch. Ivanov d,e,f \\ ${ }^{a}$ Department of Physics, Faculty of Civil Engineering, University of Rijeka, \\ Rijeka, Croatia \\ ${ }^{\mathrm{b}}$ Zagreb School of Economics and Management, Zagreb, Croatia \\ ${ }^{\mathrm{c}}$ Department of Physics, Faculty of Science, University of Zagreb, Zagreb, Croatia \\ ${ }^{\mathrm{d}}$ Center for Polymer Studies and Department of Physics, Boston University, \\ Boston, MA 02215 \\ e Division of Sleep Medicine, Brigham and Woman's Hospital, Harvard Medical \\ School, Boston, MA 02115 \\ ${ }^{\mathrm{f}}$ Institute of Solid State Physics, Bulgarian Academy of Sciences, Sofia, Bulgaria
}

\begin{abstract}
We investigate how simultaneously recorded long-range power-law correlated multivariate signals cross-correlate. To this end we introduce a two-component ARFIMA stochastic process and a two-component FIARCH process to generate coupled fractal signals with long-range power-law correlations which are at the same time longrange cross-correlated. We study how the degree of cross-correlations between these signals depends on the scaling exponents characterizing the fractal correlations in each signal and on the coupling between the signals. Our findings have relevance when studying parallel outputs of multiple-component of physical, physiological and social systems.
\end{abstract}

Many empirical data are characterized by long-range power-law auto-correlations as well as by long-range cross-correlations. Such scale-invariant organization in both auto-correlations and cross-correlations can be observed either for the data variables or their absolute values $[1,2,3,4,5,6,7,8,9]$.

Scale-invariant power-law auto-correlations in stochastic variables can be modeled by the fractionally autoregressive integrated moving-average process (ARFIMA) [10,11]: 


$$
x_{t}=\sum_{n=1}^{\infty} a_{n}(d) x_{t-n}+\epsilon_{t},
$$

where $d \in(-0.5,0.5)$ is a scaling parameter, $\epsilon_{t}$ denotes independent and identically distributed (i.i.d.) Gaussian variables with $\left\langle\epsilon_{t}\right\rangle=0$ and $\left\langle\epsilon_{t}^{2}\right\rangle=1$, $a_{n}(d)$ are the weights defined by $a_{n}(d)=d \Gamma(n-d) /(\Gamma(1-d) \Gamma(n+1))$, where $\Gamma$ denotes the Gamma function and $n$ is the time scale. We denote the autocorrelation function for $x_{t}$ as $A\left(x_{t}, x_{t-n}\right) \equiv A(n)$. For $d=0$ the generated variable $x_{t}$ becomes random.

To account for power-law cross-correlations between two variables $x_{t}$ and $y_{t}$, where each variable is itself power-law auto-correlated, we propose a twocomponent ARFIMA stochastic process defined by two stochastic variables $x_{t}$ and $y_{t}$. Each of these variables at any time depends not only on its own past values but also on past values of the other variable:

$$
\begin{aligned}
x_{t} & =\left[W X_{t}+(1-W) Y_{t}\right]+\epsilon_{t}, \\
y_{t} & =\left[(1-W) X_{t}+W Y_{t}\right]+\tilde{\epsilon}_{t}, \\
X_{t} & =\sum_{n=1}^{\infty} a_{n}\left(d_{1}\right) x_{t-n}, \\
Y_{t} & =\sum_{n=1}^{\infty} a_{n}\left(d_{2}\right) y_{t-n},
\end{aligned}
$$

where $\epsilon_{t}$ and $\tilde{\epsilon}_{t}$ denote i.i.d. Gaussian variables with $\left\langle\epsilon_{t}\right\rangle=\left\langle\tilde{\epsilon}_{t}\right\rangle=0$ and $\left\langle\epsilon_{t}^{2}\right\rangle=\left\langle\tilde{\epsilon}_{t}^{2}\right\rangle=1, a_{n}\left(d_{1}\right)$ and $a_{n}\left(d_{2}\right)$ are the weights defined in Eq. (1) through the scaling parameters $d_{1}$ and $d_{2}\left(0 \leq d_{1,2}<0.5\right)$, and $W$ is a free parameter controlling the coupling strength between $x_{t}$ and $y_{t}(0.5 \leq W \leq 1)$. We denote the cross-correlation function between $x_{t}$ and $y_{t}$ as $C\left(x_{t}, y_{t-n}\right) \equiv C(n)$. For different values of $W$ a different degree of cross-correlation between the variables $x_{t}$ and $y_{t}$ is observed. For example, for the case when $W=1$, the process defined in Eqs. (2a)-(2d) reduces to two decoupled ARFIMA processes defined in Eq. (11). Thus, when $W=1$ the long-range cross-correlations between $x_{t}$ and $y_{t}$ vanish, while both $x_{t}$ and $y_{t}$ remain long-range power-law auto-correlated.

In Fig. 1(a) we show segments of the time series $x_{t}$ and $y_{t}$ generated by the process defined in Eq. (2a)-(2d) with parameters $W=0.8$ and $d_{1}=d_{2}=0.4$. Both variables exhibit a very similar comovement. In Fig. 1(b) we show the auto-correlation functions $A(n)$ for $x_{t}$ and $y_{t}$, as well as the cross-correlation function $C\left(x_{t}, y_{t-n}\right) \equiv C(n)$. These three curves practically overlap [Fig. 1(b), three top curves]. We also show the same correlation functions for $W=0.8$ and $d_{1}=d_{2}=0.3$ [Fig. 1(b), three bottom curves]. Generally, when the coupling parameter $W$ is kept fixed, the stochastic process we introduce in Eq. (2) generates stronger cross-correlations for larger values of the scaling 

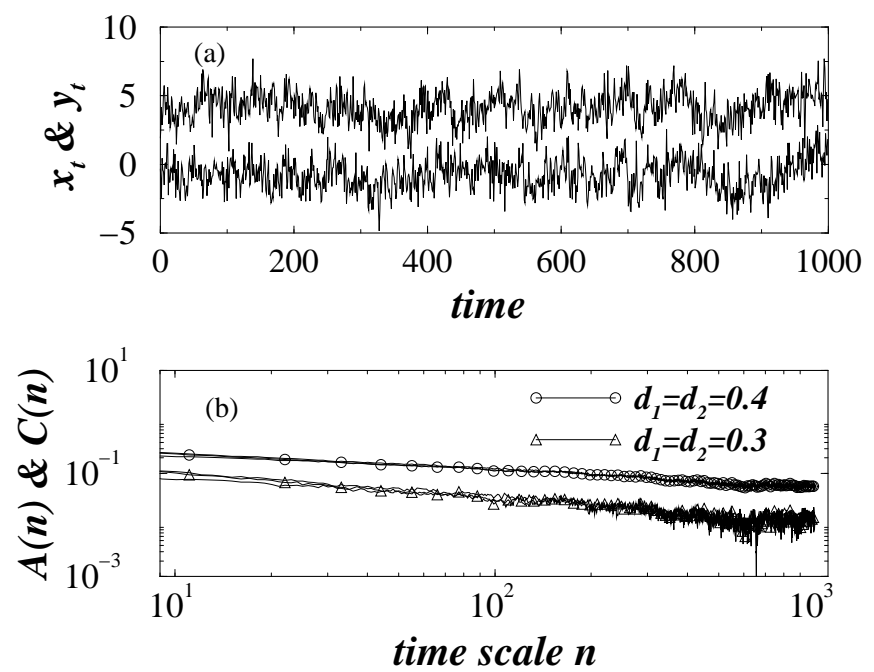

Fig. 1. (a) Time series $x_{t}$ and $y_{t}$ for the process defined in Eqs.(2a)-(WSigy) where $W=0.8$ and $d_{1}=d_{2}=0.4$. The time series $x_{t}$ is vertically shifted for clarity. Both $x_{t}$ and $y_{t}$ exhibit apparent comovement, indicating a high degree of cross-correlation. (b) Log-log plots of the auto-correlation functions $A(n)$ for $x_{t}$ and $y_{t}$, and their cross-correlation function $C(n)$ for the two-component ARFIMA process with $W=0.8$ and $d_{1}=d_{2}=0.4$ (top three curves), and with $W=0.8$ and $d_{1}=d_{2}=0.3$ (bottom three curves). For decreasing values of the scaling parameters $d_{1}$ and $d_{2}$ both the auto-correlations and cross-correlations decrease, leading to smaller values of $A(n)$ and $C(n)$.

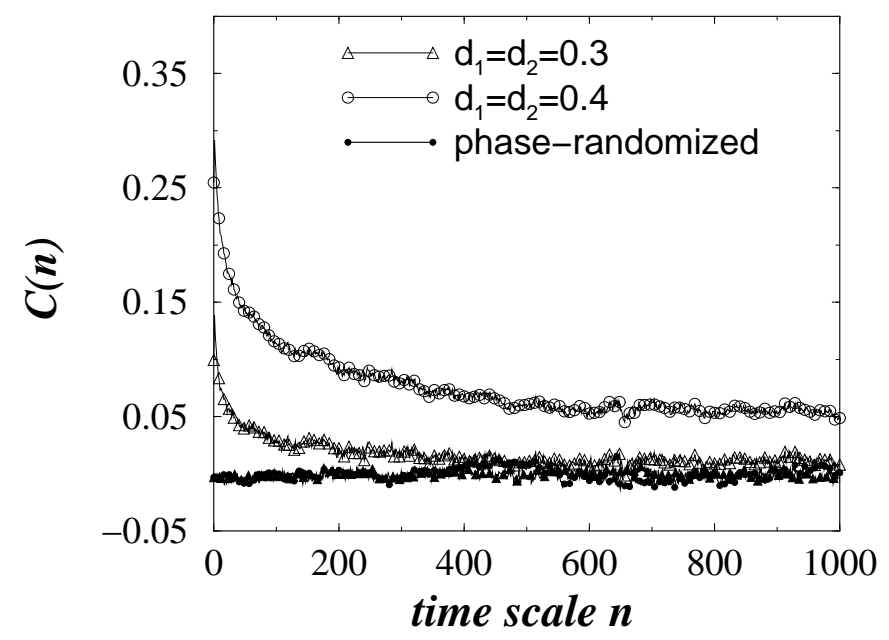

Fig. 2. Cross-correlation function $C(n)$ before Fourier phase-randomization procedure for the time series $x_{t}$ and $y_{t}$ shown in Fig. 1 (open symbols). After Fourier phase randomization of $x_{t}$ and $y_{t}$ the cross-correlation function virtually disappears (filled symbols) for any value of $d_{1}$ and $d_{2}$.

parameters $d_{1}$ and $d_{2}$.

Motivated by the fact that for linear processes the auto-correlation function does not change under randomization of the Fourier phase [13.14], we next test 


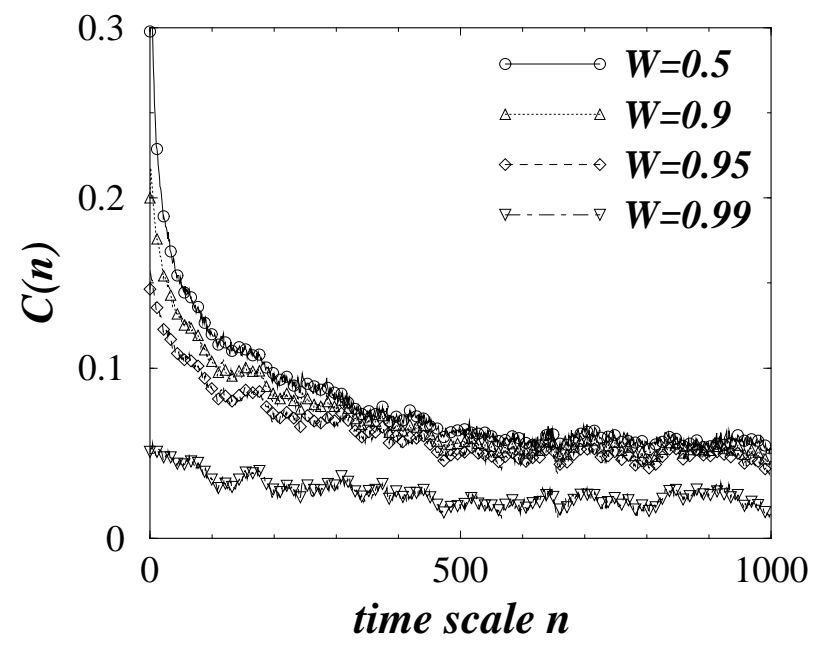

Fig. 3. Cross-correlation function $C(n)$ between time series $x_{t}$ and $y_{t}$ generated by the process in Eqs.(2) for varying values of $W$ and $d_{1}=d_{2}=0.4$. The cross-correlation function has highest values for $W=0.5$, and tends to zero for $W$ approaching 1. When $W=1, x_{t}$ and $y_{t}$ become two decoupled ARFIMA processes.

how this phase-randomization procedure affects the degree of cross-correlation between $x_{t}$ and $y_{t}$. First, we perform a Fourier transform of the original time series, e.g. $x_{t}$, preserving the Fourier amplitudes but randomizing the Fourier phases. Then, we perform an inverse Fourier transform and obtain a surrogate (linearized) time series $\tilde{x}_{t}$. Applying this phase-randomization procedure to both time series $x_{t}$ and $y_{t}$ generated by the two-component ARFIMA process in Eq. (2), we calculate the two auto-correlation functions for $\tilde{x}_{t}$ and $\tilde{y}_{t}$, as well as their cross-correlation function $C\left(\tilde{x}_{t}, \tilde{y}_{t-n}\right)$. As expected, the autocorrelation functions remain unchanged after Fourier phase randomization, but the cross-correlation function $C\left(\tilde{x}_{t}, \tilde{y}_{t-n}\right)$ completely vanishes [Fig. 2].

Next, we investigate the case when the scaling parameters $d_{1}$ and $d_{2}$ are fixed, while the coupling parameter $W$ varies. In Fig. 3, we show how the cross-correlation function changes for different values of $W$ and for fixed $d_{1}=d_{2}=0.4$. The closer the value of the parameter $W$ to 1 , the weaker the cross-correlations ( $W=1$ corresponds to the case of two decoupled ARFIMA processes).

Next we analyze how the degree of power-law auto-correlations changes when varying the parameters $W, d_{1}$, and $d_{2}$ in Eqs. (2a) $-(2 \mathrm{~d})$. To quantify the autocorrelations we employ the detrended fluctuations analysis (DFA) method. We estimate the rms fluctuation function $F(n)$ for different time scales $n$ [15, 16, 17, 18, 19]. A power-law dependence of $F(n)$ on the time scale $n$ $F(n) \propto n^{\alpha}$, where $\alpha$ is the correlation exponent - indicates presence of power law auto-correlations. In Fig. 4, we show the DFA scaling curves obtained for $x_{t}$ and $y_{t}$ generated by the two-component ARFIMA process in Eqs. (2a)-(2d), where $d_{1}=0.4$ and $d_{2}=0.1$, and the coupling parameter $W$ varies. For $W=1$ 




Fig. 4. DFA scaling curves for the time series $x_{t}$ and $y_{t}$ generated by the twocomponent ARFIMA process in Eqs. (2a)-(2d), where $d_{1}=0.4$ and $d_{2}=0.1$. For $W=1, x_{t}$ and $y_{t}$ are decoupled and thus not cross-correlated, and $x_{t}$ behaves as the ARFIMA process in Eq. (1) defined only by the scaling parameter $d_{1}$, while $y_{t}$ becomes a separate ARFIMA process defined only by the scaling parameter $d_{2}$. For $W \neq 1$, the scaling properties of $x_{t}$ depend on both parameters $d_{1}$ and $d_{2}$. When $W=0.5$, the DFA correlation exponent $\alpha$ for $x_{t}$ becomes equal to the DFA correlation exponent for $y_{t}$. The DFA exponent for $\left|y_{t}\right|$ does not depend on $W$.

the processes $x_{t}$ and $y_{t}$ are decoupled and thus not cross-correlated. In this case, $x_{t}$ behaves as a power-law auto-correlated ARFIMA process controlled by only the scaling parameter $d_{1}$, with the DFA correlation exponent equals $\alpha=0.5+d_{1}=0.9$. Similarly, $y_{t}$ becomes a separate ARFIMA process (decoupled from $x_{t}$ ) which is controlled only by the scaling parameter $d_{2}$, where $\alpha=0.5+d_{2}=0.6$. We find that with decreasing value of $W$ (from 1 to 0.5 ), $x_{t}$ becomes a mixture of two ARFIMA processes and the DFA correlation exponent $\alpha$ gradually decreases towards $\alpha=0.6$ corresponding to the $y_{t}$ process, controlled by parameter $d_{2}=0.1$. In contrast to $x_{t}$, for the process $y_{t}$ the DFA correlation exponent $\alpha$ virtually does not change with varying the coupling parameter $W$.

We next consider a separate stochastic process which generates simultaneously two time series with power-law auto-correlated absolute values of their variables and long-range cross-correlations between these absolute values. Powerlaw auto-correlations in the absolute values of the stochastic variables can be modeled by the Fractionally Integrated ARCH (FIARCH) process [20]12]:

$$
\begin{aligned}
x_{t} & =\sigma_{t} \epsilon_{t} \\
\sigma_{t} & =\sum_{n=1}^{\infty} a_{n}(d) \frac{\left|x_{t-n}\right|}{\mu_{x}},
\end{aligned}
$$


where $\epsilon_{t}$ denotes an i.i.d. Gaussian variable with $\left\langle\epsilon_{t}\right\rangle=0$ and $\left\langle\epsilon_{t}^{2}\right\rangle=1$, and $0<d<1 / 2$ and $\mu_{x}=\left\langle\left|x_{t}\right|\right\rangle$. The sum of the weights $a_{n}(d)$ satisfies $\sum_{n=1}^{\infty} \frac{d \Gamma(n-d)}{\Gamma(1-d) \Gamma(n+1)}=1$, yielding $\left\langle\sigma_{t}\right\rangle=1$. While for the time series $x_{t}$ generated by Eq. (1) the autocorrelation function $A\left(x_{t}, x_{t-n}\right)$ is zero for all time scales $n$, for the absolute values $\left|x_{t}\right|$ the auto-correlation function is $A\left(\left|x_{t}\right|,\left|x_{t-n}\right|\right)=\Gamma(1-d) \Gamma(n+d) /(\Gamma(d) \Gamma(n+1-d))$, which for $n>>1$ converges to the power law $A(n) \sim n^{-1+2 d}$.

To account for power-law cross-correlations between the absolute values of two variables, where the absolute values of each variable are simultaneously powerlaw auto-correlated, we have previously introduced [21] a two-component FIARCH process with scaling parameters $d_{1}$ and $d_{2}$ :

$$
\begin{aligned}
x_{t} & =\left[W \sigma_{x t}+(1-W) \sigma_{y t}\right] \epsilon_{t} \\
y_{t} & =\left[(1-W) \sigma_{x t}+W \sigma_{y t}\right] \tilde{\epsilon}_{t} \\
\sigma_{x t} & =\sum_{n=1}^{\infty} \frac{d_{1} \Gamma\left(n-d_{1}\right)}{\Gamma\left(1-d_{1}\right) \Gamma(n+1)} \frac{\left|x_{t-n}\right|}{\mu_{x}} \\
\sigma_{y t} & =\sum_{n=1}^{\infty} \frac{d_{2} \Gamma\left(n-d_{2}\right)}{\Gamma\left(1-d_{2}\right) \Gamma(n+1)} \frac{\left|y_{t-n}\right|}{\mu_{y}} .
\end{aligned}
$$

where $\epsilon_{t}$ and $\tilde{\epsilon}_{t}$ are i.i.d. variables with $\left\langle\epsilon_{t}\right\rangle=\left\langle\tilde{\epsilon}_{t}\right\rangle=0$ and $\left\langle\tilde{\epsilon}_{t}^{2}\right\rangle=\left\langle\epsilon_{t}^{2}\right\rangle=1$, $W$ is the coupling parameter controlling the degree of cross-correlations, and $\mu_{x}=\left\langle\left|x_{t}\right|\right\rangle$ and $\mu_{y}=\left\langle\left|y_{t}\right|\right\rangle$.

Note, that each of the variables is controlled by a composite volatility - e.g. for $x_{t}$ the composite volatility is $W_{1} \sigma_{x t}+\left(1-W_{1}\right) \sigma_{y t}$ [Eq. (4a)] - that is a combination of two FIARCH volatilities $\sigma_{x t}$ and $\sigma_{x t}[\mathrm{Eq}$. (3b)]. Stability of the FIARCH process is achieved through the condition $\left\langle\sigma_{t}\right\rangle=1$. To retain stability for the two-component FIARCH process in Eq. (4), the average values of the composite volatilities $W \sigma_{x t}+(1-W) \sigma_{y t}$ and $(1-W) \sigma_{x t}+W \sigma_{y t}$ in Eqs. (4a)-(4b) should be 1. For $W=1$ the process in Eqs. (4a)-(4d ) reduces to two decoupled FIARCH process as defined in Eqs. (3b)-(3b), and thus $\left|x_{t}\right|$ and $\left|y_{t}\right|$ are not cross-correlated.

In Ref. [21] we have analyzed the cross-correlation functions between $\left|x_{t}\right|$ and $\left|y_{t}\right|$ for the process defined in Eqs. (4a)-(4d) for varying values of the parameters $W, d_{1}$, and $d_{2}$.

Finally, we analyze how the auto-correlations in the absolute values change when varying the parameters $W, d_{1}$, and $d_{2}$. In Fig. 5 , we show the DFA scaling curves for $d_{1}=0.4$ and $d_{2}=0.1$, and for varying $W$. For $W=1$, the time series $x_{t}$ and $y_{t}$ are decoupled and so not cross-correlated. In this case, $x_{t}$ is a FIARCH process controlled only by the scaling parameter $d_{1}$, and exhibits long-range power-law auto-correlations characterized by a DFA 


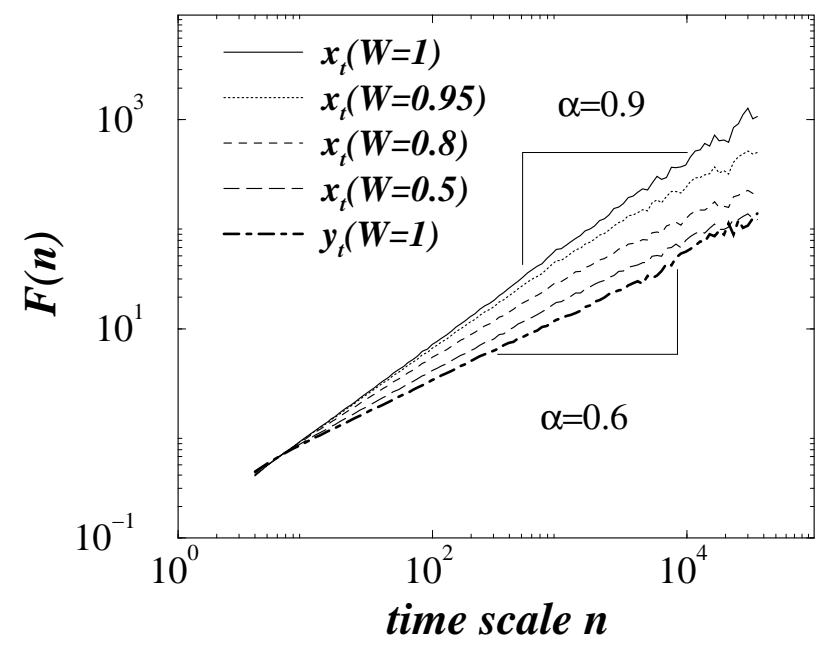

Fig. 5. DFA scaling curves of the time series $\left|x_{t}\right|$ and $\left|y_{t}\right|$ generated by the twocomponent FIARCH process in Eqs. (4a)-(4d), where $d_{1}=0.4$ and $d_{2}=0.1$. For $W=1,\left|x_{t}\right|$ and $\left|y_{t}\right|$ are decoupled and thus are not cross-correlated. In this case, $x_{t}$ becomes a separate FIARCH process as defined in Eqs. (3), and the auto-correlation properties of $x_{t}$ depend only on the scaling parameter $d_{1}$, while $y_{t}$ is another FIARCH process with auto-correlation properties depending only on the parameter $d_{2}$. For $W \neq 1$, the scaling properties of $x_{t}$ depend on both parameters $d_{1}$ and $d_{2}$. When $W=0.5$, the DFA correlation exponent $\alpha$ for $\left|x_{t}\right|$ becomes equal to the DFA correlation exponent for $\left|y_{t}\right|$. Note that the DFA exponent for $\left|y_{t}\right|$ does not depend on $W$.

correlation exponent $\alpha=0.5+d_{1}=0.9$. Similarly, $y_{t}$ is another FIARCH process controlled only by $d_{2}$, and characterized by $\alpha=0.5+d_{2}=0.6$. We find that with decreasing value of $W$ (from 1 to 0 ), $x_{t}$ is controlled by both parameters $d_{1}$ and $d_{2}$, and the DFA exponent $\alpha$ gradually decreases towards the value $\alpha=0.6$. At the same time, the process $y_{t}$ which is controlled only by the parameter $d_{2}=0.1$ is also characterized by $\alpha=0.6$, regardless of the values of $W$.

The presented modeling approach and findings may have relevance when quantifying cross-correlations in simultaneously recorded multivariate time series of fractal nature. This problem is pertinent to multiple component physical [22,23,24, physiological, social and financial systems.

We thank the Ministry of Science of Croatia, NIH (Grant HL071972) and NSF for financial support.

\section{References}

[1] C. C. Ying, Econometrica 34, (1966) 676.

[2] R. L. Crouch, Financial Analystss Journal 26 (1970) 104. 
[3] G. Tauchen and M. Pitts, Econometrica 51, (1983) 485.

[4] J. Karpoff, Journal of Financial and Quantitative Analysis 22 (1987) 109.

[5] R. Gallant, P. Rossi, and G. Tauchen, Review of Financial Studies 5 (1992) 199.

[6] J. Campbell, A. W. Lo and A. MacKinlay, The Econometrics of Financial Markets Princeton NJ: Princeton University Press (1997).

[7] V. Plerou et al., Quantitative Finance 1 (2001) 262.

[8] P. Gopikrishnan et al., Physical Review E 62 (2000) 4493.

[9] B. LeBaron, W. B. Arthur, and R. Palmer, Journal of Economic Dynamic \& Control 23, (1999) 1487.

[10] C. W. J. Granger and R. Joyeux, J. Time Series Analysis 1, (1980) 15.

[11] J. Hosking, Biometrika 68, (1981) 165.

[12] B. Podobnik et al., Phys. Rev. E 72, (2005) 026121.

[13] J. Theiler et al., Physica D 58, (1992) 77.

[14] Y. Ashkenazy et al., Physica A 323, (2003) 19.

[15] C.-K. Peng et al., Phys. Rev. E 49, (1994) 1685.

[16] K. Hu et al., Phys. Rev. E 64(1) (2001) 011114(19).

[17] Z. Chen et al., Phys. Rev. E 65(4) (2002) 041107(15).

[18] Z. Chen et al., Phys. Rev. E 71(1) (2005) 011104(11).

[19] L. Xu et al., Phys. Rev. E 71(5) (2005) 051101(14).

[20] C. W. J. Granger and Z. Ding Annales d'Economie et de Statistique 40 (1995) 67.

[21] B. Podobnik et al., Eur. Phys. J. B 56, (2007) 47.

[22] G. Nugent-Glandorf et al., Phys. Rev. Lett. 87(19) (2001) 193002.

[23] O. A. Godin, Phys. Rev. Lett. 97 (2006) 054301.

[24] M. Campilo and A. Paul, Science 299 (2003) 547. 